\title{
Trials clinici nella nefrolitiasi: di che cosa abbiamo bisogno? Presente e futuro della ricerca clinica
}

\author{
L. Borghi ${ }^{1}$, A. Nouvenne ${ }^{1}$, B. Prati ${ }^{1}$, F. Allegri ${ }^{1}$, A. Guerra ${ }^{1}$, U. Maggiore ${ }^{2}$, \\ T. Meschi ${ }^{1}$ \\ ${ }^{1}$ Dipartimento di Scienze Cliniche, Università di Parma \\ ${ }^{2}$ Dipartimento di Medicina Interna, Nefrologia e Scienze della Prevenzione, Università di Parma
}

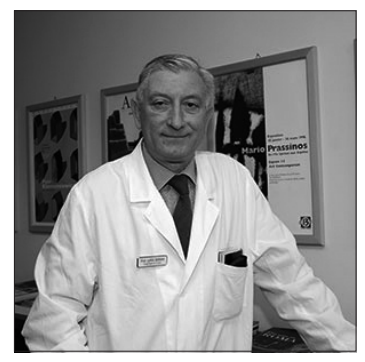

\section{Introduzione}

Nonostante l'importante morbilità legata alla elevata prevalenza della calcolosi renale nella popolazione generale, esistono relativamente pochi studi clinici sul suo trattamento.

L. Borghi

La sperimentazione clinica è lo strumento più affidabile a nostra disposizione per valutare l'efficacia di un trattamento. La qualità delle prove scientifiche fornite da uno studio clinico dipende dalla specificità del contesto in cui l'intervento è testato, cioè dalle sue indicazioni, dalle caratteristiche e dal grado di compliance raggiunto (1).

Il trial clinico è uno studio prospettico effettuato per confrontare l'effetto di uno o più interventi nei confronti di un controllo (2). Alla valutazione basale è necessario che il gruppo di controllo sia simile al gruppo di intervento: solo così sarà possibile ragionevolmente attribuire le differenze di risultato all'effetto dell'intervento. La randomizzazione è il modo preferito di assegnare i partecipanti al gruppo di controllo o al gruppo di intervento; essa tende, nel lungo periodo, a bilanciare equamente tutti i fattori prognostici, compresi quelli non misurati, non misurabili e/o sconosciuti.

Sia il ricercatore che il partecipante dovrebbero agire "in cieco" nei confronti del trattamento anche per minimizzare il rischio che siano prescritti trattamenti compensativi in modo diverso nei gruppi. Ovviamente, men- tre il "doppio cieco" è facilmente utilizzabile per testare farmaci, la sua applicazione è praticamente impossibile quando l'obiettivo è valutare l'effetto di un intervento dietetico.

\section{Scelta della popolazione in studio}

Prima di iniziare il trial è indispensabile specificare la categoria di pazienti da studiare e la strategia da utilizzare per il reclutamento (3).

I pazienti in esame sono definiti dai criteri di inclusione ed esclusione. Questi criteri sono scelti in modo da selezionare i pazienti che con maggiore probabilità potrebbero trarre beneficio dal trattamento. Ad esempio, un trattamento dietetico sarebbe meno efficace in caso di malattie ereditarie come la cistinuria.

Anche la strategia pratica di reclutamento è importante. Per esempio, l'arruolamento effettuato in centri di II o III livello piuttosto che dai medici di Medicina Generale tende a selezionare pazienti più gravi e più motivati; esiste, nella Medicina Generale, un unico RCT in doppio cieco sulla nefrolitiasi che, nonostante un bacino di utenza di 17.000 abitanti, è stato in grado di includere solo 50 soggetti, seguiti per una media di 40 mesi (4).

La popolazione in studio dovrebbe essere la più omogenea possibile. È obbligatoria una caratterizzazione precisa del fenotipo del calcolo. Questo è spesso difficile a causa della incostante disponibilità dell'analisi della composizione del calcolo. Nella nefrolitiasi calcica, l'analisi della composizione del calcolo permette di distinguere 
tra calcoli di calcio-ossalato, calcio-fosfato e misti con percentuali diverse, perché questi calcoli si differenziano per storia naturale, gravità, incidenza di recidive e risposta al trattamento. Per questo motivo diversi studi pubblicati non sono facilmente confrontabili (5-7). Inoltre, le popolazioni devono essere omogenee per quanto riguarda le alterazioni metaboliche (ipercalciuria, ipocitraturia ecc.). Poiché queste anomalie sono note per aumentare il rischio di nefrolitiasi, in diversi studi i pazienti sono selezionati sulla base di esse (5, 7-12). Un'eccezione a questa regola è il caso in cui un trattamento può essere ugualmente efficace su due o più tipi di calcolo. Anche molti altri aspetti, come la gravità della malattia, gli interventi chirurgici, il numero di calcoli ecc. devono essere valutati con precisione.

\section{Stima della dimensione del campione e presupposti}

Ogni sperimentazione clinica deve arruolare un numero sufficiente di partecipanti per raggiungere una potenza statistica adeguata. Il potere è la probabilità che il trial rilevi una vera differenza tra i trattamenti, cioè sia statisticamente significativo. La potenza dipende dalla grandezza della differenza tra i trattamenti, dalla variabilità della risposta, e dall'analisi statistica in uso (13) Comunemente, una dimensione del campione è scelta in modo tale da ottenere una potenza da 80 a $95 \%$. Il complemento del potere è la probabilità di un risultato falso negativo. Per esempio, un campione in cui si raggiunge una potenza dell' $80 \%$, ha un rischio del $20 \%$ di un risultato falsamente negativo.

Uno studio clinico sulla recidiva litiasica dovrebbe durare almeno tre anni, preferibilmente cinque, poiché quasi ogni trattamento profilattico mostra il suo effetto significativo in media dopo 2 anni. Infatti, per fattori in gran parte sconosciuti, i trattamenti profilattici più efficaci descritti in letteratura sono in grado di indurre una rapida (settimane o mesi) riduzione dei fattori urinari di rischio litogeno, mentre la maggiore riduzione del tasso di recidiva si verifica in seguito (> 2 anni) (5-9, 14-17). Per calcolare la potenza, lo sperimentatore deve specificare in anticipo la presunta differenza di risposta tra i gruppi intervento e controllo. L'effetto dell'intervento di solito è dedotto da studi precedentemente pubblicati. Spesso, però, sono disponibili solo le informazioni riguardanti la prognosi dei pazienti con il trattamento standard. In questo caso, può essere considerata clinicamente significativa anche una differenza minima.

A volte, si può valutare se un trattamento è equivalente o, semplicemente, non inferiore ad un altro. I trials di equivalenza e di non inferiorità sono spesso previsti ogni volta che il trattamento standard sia costoso o poco gradito ai pazienti, rendendo così preferibile un trattamento altrettanto efficace ma più semplice.

La Tabella I elenca tutti gli studi di intervento randomizzati pubblicati sulla nefrolitiasi e i dati che potrebbero essere alla base per il calcolo della dimensione del campione.

\section{Valutazione basale}

Prima di iniziare l'intervento, tutte le caratteristiche di base dovrebbero essere misurate in tutti i partecipanti. Nei gruppi randomizzati questo ci permette di valutare il successo del processo di randomizzazione e in che modo i risultati dello studio sono confrontabili con quelli provenienti da altri studi.

Individuare gli squilibri non inficia i risultati, ma rende più complicata la loro interpretazione. Di solito le tecniche di regressione multipla o stratificazione sono utilizzate per correggere i risultati, quando sono presenti differenze di base. Vale la pena notare che $\mathrm{i}$ test statistici che confrontano i gruppi randomizzati al basale (come riportato in diversi articoli di ricerca) sono privi di significato, dal momento che la randomizzazione rende l'ipotesi "null" vera per definizione. È essenziale che la definizione di "baseline" sia uniforme per l'intera popolazione in studio e che le caratteristiche di base rispecchino il più strettamente possibile le condizioni cliniche al momento della randomizzazione.

La Tabella II mostra l'impostazione di base ideale per eseguire correttamente un RCT per valutare l'effetto di un intervento sul tasso di recidiva litiasica. Come detto, la caratterizzazione della composizione dei calcoli è fondamentale. Al contrario, la misurazione dei fattori urinari di rischio litogeno non è obbligatoria se l'endpoint primario è la "recidiva litiasica". In questo contesto, la conoscenza della composizione urinaria è utile a sostenere le conclusioni relative all'endpoint primario, ma non è essenziale per valutare l'efficacia dell'intervento.

\section{Intervento e farmaci concomitanti}

La Tabella III mostra i principali RCT pubblicati con la "recidiva", come obiettivo primario, classificati per la composizione del calcolo.

Gli interventi farmacologici o dietetici sono possibili se 
TABELLA I - DATI NECESSARI PER UN CALCOLO DELLA DIMENSIONE DEL CAMPIONE SULLA BASE DI TUTTI GLI INTERVENTIONAL RCT PUBBLICATI

\begin{tabular}{|c|c|c|c|c|c|}
\hline Obiettivo & Popolazione studiata & Intervento & Central tendency & Variabilità & Bibliografia \\
\hline $\begin{array}{l}\text { Escrezione di ossalato } \\
\text { urinario }\end{array}$ & $\begin{array}{l}\text { Produttori di calcoli di } \\
\mathrm{CaOx} \text { con iperossaluria }\end{array}$ & Probiotici (Oxadrop) & $52.6 \mathrm{mg}$ & IQR da 44.6 a 68 & $\begin{array}{l}\text { Goldfarb DS, JASN } \\
2007 ; 2: 745-749\end{array}$ \\
\hline & Maschi e Femmine & & & & \\
\hline $\begin{array}{l}\text { Fattori di rischio per calcoli } \\
\text { urinari }\end{array}$ & $\begin{array}{l}\text { Soggetti sani a riposo a } \\
\text { letto in dieta metabolica } \\
\text { Maschi e Femmine }\end{array}$ & KMg Citrato & $\begin{array}{l}\text { Ca } 222 \mathrm{mg} \\
\mathrm{K} 82 \mathrm{mEq} \\
\mathrm{Mg} 165 \mathrm{mg} \\
\mathrm{Cit} 976 \mathrm{mg} \\
\mathrm{pH} 6.56 \\
\text { RSR CaOx } 3.37 \\
\text { RSR brush } 1.82 \\
\text { Acido Urico } \\
\text { Indissociato } 70 \mathrm{mg}\end{array}$ & $\begin{array}{l}\text { SD } 71 \\
\text { SD } 11 \\
\text { SD } 45 \\
\text { SD } 330 \\
\text { SD } 0.41 \\
\text { SD } 1.50 \\
\text { SD. } 01 \\
\text { SD } 91\end{array}$ & $\begin{array}{l}\text { Zerwekh JE, } \\
\text { J Urol 2007; } \\
\text { 177: 2179-2184 }\end{array}$ \\
\hline $\begin{array}{l}\text { Fattori di rischio per calcoli } \\
\text { urinari }\end{array}$ & $\begin{array}{l}\text { Soggetti sani e produt- } \\
\text { tori di calcoli di } \mathrm{CaOx} \text { in } \\
\text { dieta metabolica } \\
\text { Maschi e Femmine }\end{array}$ & Succo d'arancia & $\begin{array}{l}\text { Cit } 1000 \mathrm{mg} \\
\text { NAE } 17.3 \\
\mathrm{Mg} 108 \mathrm{mg} \\
\mathrm{K} 85 \mathrm{mEq} \\
\mathrm{Amm} 23 \mathrm{mEq} \\
\mathrm{Ox} 35 \mathrm{mg} \\
\mathrm{RS} \mathrm{CaOx} 3.71 \\
\text { RS brush } 0.82\end{array}$ & $\begin{array}{l}\text { Range } 450-1400 \\
\text { SD } 20.8 \\
\text { SD } 28 \\
\text { SD } 21 \\
\text { SD } 8 \\
\text { SD } 6 \\
\text { SD } 1.58 \\
\text { SD } 0.56\end{array}$ & $\begin{array}{l}\text { Odvina CV, } \\
\text { JASN 2006; 1: } 1269 \text { - } \\
1274\end{array}$ \\
\hline $\begin{array}{l}\text { Escrezione, assorbimento, } \\
\text { sintesi endogena di ossalato }\end{array}$ & $\begin{array}{l}\text { Soggetti sani e produttori } \\
\text { di calcoli di } \mathrm{CaOx} \\
\text { in dieta metabolica } \\
\text { Maschi e Femmine }\end{array}$ & Acido Ascorbico & $\begin{array}{l}\text { Ox ur } 51.2 \mathrm{mg} \\
\text { Ox assorbimento \% } 10.5 \\
\text { Ox endogeno } 49 \mathrm{mg}\end{array}$ & $\begin{array}{l}\text { SD } 12.5 \\
\text { SD } 3.2 \\
\text { SD } 12\end{array}$ & $\begin{array}{l}\text { Massey LK, } \\
\text { J Nutr } \\
\text { 2005; 135: } 1673-1677\end{array}$ \\
\hline $\begin{array}{l}\text { Calcio e Ossalato Urinari, } \\
\text { Tiselius Risk Index (TRI) }\end{array}$ & $\begin{array}{l}\text { Soggetti sani in dieta } \\
\text { metabolica } \\
\text { Maschi }\end{array}$ & Ca Carbonato & $\begin{array}{l}\text { Ox } 11.7 \mathrm{mg} \\
\text { Cit } 159 \mathrm{mg} \\
\text { Ca } 207 \mathrm{mg} \\
\text { TRI } 0.57\end{array}$ & $\begin{array}{l}\text { SD } 4.5 \\
\text { SD } 109 \\
\text { SD } 104 \\
\text { SD } 0.22\end{array}$ & $\begin{array}{l}\text { Domrongkitchaiporn S, } \\
\text { Kidney Int 2004; 65: } \\
\text { 1835-1841 }\end{array}$ \\
\hline $\begin{array}{l}\text { Fattori di rischio per calcoli } \\
\text { urinari }\end{array}$ & $\begin{array}{l}\text { Soggetti sani in dieta } \\
\text { metabolica } \\
\text { Maschi e Femmine }\end{array}$ & Dieta povera di Ca & $\begin{array}{l}\text { Ca } 148 \mathrm{mg} \\
\text { Ox } 25 \mathrm{mg} \\
\text { Vol } 1814 \mathrm{~mL} \\
\text { pH } 6.25 \\
\text { RS CaOx } 4.3 \\
\text { Cit } 849 \mathrm{mg}\end{array}$ & $\begin{array}{l}\text { SD } 55 \\
\text { SD } 7 \\
\text { SD } 626 \\
\text { SD } 0.31 \\
\text { SD } 2.2 \\
\text { SD } 299\end{array}$ & $\begin{array}{l}\text { Heller HJ, } \\
\text { J Urol 2003; 169: 470- } \\
474\end{array}$ \\
\hline $\begin{array}{l}\text { Fattori di rischio per calcoli } \\
\text { urinari }\end{array}$ & $\begin{array}{l}\text { Soggetti sani } \\
\text { Maschi }\end{array}$ & Succo di mirtillo & $\begin{array}{l}\text { Ca } 121 \mathrm{mg} \\
\text { Ox } 9.9 \mathrm{mg} \\
\text { Cit } 714 \mathrm{mg} \\
\text { Vol } 1805 \mathrm{mLí} \\
\text { Limite di metastabilità } \\
0.007 \\
\text { RS CaOx } 1.46 \\
\text { RS ac urico } 0.96 \\
\text { RS brushite } 0.68\end{array}$ & $\begin{array}{l}\text { SD } 44 \\
\text { SD } 1.8 \\
\text { SD } 129 \\
\text { SD } 176.53 \\
\text { SD } 0.45 \\
\text { SD } 9.16 \\
\text { SD } 7.48 \\
\text { SD } 7.48\end{array}$ & $\begin{array}{l}\text { McHarg T, } \\
\text { BJU Int 2003; 92: 765- } \\
768\end{array}$ \\
\hline
\end{tabular}


TABELLA I - DATI NECESSARI PER UN CALCOLO DELLA DIMENSIONE DEL CAMPIONE SULLA BASE DI TUTTI GLI INTERVENTIONAL RCT PUBBLICATI

\begin{tabular}{|c|c|c|c|c|c|}
\hline Obiettivo & Popolazione studiata & Intervento & Central tendency & Variabilità & Bibliografia \\
\hline Recidiva di calcoli & $\begin{array}{l}\text { Produttori recidivi } \\
\text { di calcoli di CaOx con } \\
\text { ipercalciuria idiopatica } \\
\text { Maschi }\end{array}$ & $\begin{array}{l}\text { Dieta a contenuto } \\
\text { normale di calcio } \\
\text { e a basso contenuto } \\
\text { di proteine e di sale }\end{array}$ & $20 \%$ a 5 anni & - & $\begin{array}{l}\text { Borghi L, } \\
\text { NEJM 2002; 346: 77-84 }\end{array}$ \\
\hline Recidiva di calcoli & $\begin{array}{l}\text { Produttori di calcoli } \\
\text { di CaOx post-litotripsia } \\
\text { Maschi e Femmine }\end{array}$ & KCitrato & $0 \%$ a 1 anno & - & $\begin{array}{l}\text { Soygur T, } \\
\text { J Endourol 2002; 16: } \\
\text { 149-152 }\end{array}$ \\
\hline Recidiva di calcoli & $\begin{array}{l}\text { Produttori recidivi } \\
\text { di calcoli di } \mathrm{CaOx} \\
\text { Maschi e Femmine }\end{array}$ & $\mathrm{KMg}$ Citrato & $12.9 \%$ a 3 anni & - & $\begin{array}{l}\text { Ettinger B, } \\
\text { J Urol 1997;158: 2069- } \\
2073\end{array}$ \\
\hline Recidiva di calcoli & $\begin{array}{l}\text { Produttori di calcoli di } \\
\text { calcio (primo episodio) } \\
\text { Maschi e Femmine }\end{array}$ & Acqua & $12.1 \%$ a 5 anni & - & $\begin{array}{l}\text { Borghi L, } \\
\text { J Urol 1996; 155: 839- } \\
843\end{array}$ \\
\hline Recidiva di calcoli & $\begin{array}{l}\text { Produttori recidivi } \\
\text { di calcoli di } \mathrm{CaOx} \\
\text { Maschi e Femmine }\end{array}$ & NaKCitrato & $69 \%$ a 3 anni & - & $\begin{array}{l}\text { Hofbauer J, } \\
\text { BJU 1994; 73: 362-365 }\end{array}$ \\
\hline $\begin{array}{l}\text { Recidiva di calcoli } \\
\text { e Stone Rate }\end{array}$ & $\begin{array}{l}\text { Produttori recidivi di } \\
\text { calcoli di } \mathrm{CaOx} \text { e } \mathrm{CaP} \\
\text { con ipocitraturia } \\
\text { Maschi e Femmine }\end{array}$ & KCitrato & $\begin{array}{l}27.8 \% \text { a } 3 \text { anni } \\
\text { Stone Rate } 0.1\end{array}$ & $\begin{array}{l}- \\
\text { SD } 0.2\end{array}$ & $\begin{array}{l}\text { Barcelo P, } \\
\text { J Urol 1993;150: 1761- } \\
1764\end{array}$ \\
\hline $\begin{array}{l}\text { Recidiva di calcoli } \\
\text { e Stone Rate }\end{array}$ & $\begin{array}{l}\text { Produttori recidivi di cal- } \\
\text { coli di CaOx e di CaOx- } \\
\mathrm{CaP} \text { con ipercalciuria } \\
\text { Maschi e Femmine }\end{array}$ & $\begin{array}{l}\text { Dieta, Fluidi, Indapa- } \\
\text { mide e Allopurinolo }\end{array}$ & $\begin{array}{l}12.5 \% \text { a } 3 \text { anni } \\
\text { Stone Rate } 0.04\end{array}$ & $-\overline{S D} 0.11$ & $\begin{array}{l}\text { Borghi L, } \\
\text { J Cardiovasc Pharm } \\
\text { 1993; 22: S78-S86 }\end{array}$ \\
\hline $\begin{array}{l}\text { Recidiva di calcoli } \\
\text { e Stone Rate }\end{array}$ & $\begin{array}{l}\text { Produttori recidivi di cal- } \\
\text { coli di } \mathrm{CaOx} \text { e di CaOx- } \\
\mathrm{CaP} \text { con ipercalciuria } \\
\text { Maschi e Femmine }\end{array}$ & $\begin{array}{l}\text { Diuretici tiazidici } \\
\text { (Triclormetiazide) }\end{array}$ & $\begin{array}{l}8.3 \% \text { a } 2 \text { anni } \\
\text { Stone Rate } 0.13\end{array}$ & $\begin{array}{l}- \\
\text { SD } 0.33\end{array}$ & $\begin{array}{l}\text { Ohkawa M, } \\
\text { BJU 1992; 69: 571-576 }\end{array}$ \\
\hline $\begin{array}{l}\text { Recidiva di calcoli } \\
\text { e Stone Rate }\end{array}$ & $\begin{array}{l}\text { Produttori recidivi di } \\
\text { calcoli di calcio } \\
\text { Maschi }\end{array}$ & $\begin{array}{l}\text { Diuretici tiazidici } \\
\text { (Triclormetiazide) } \\
\text { e Allopurinolo }\end{array}$ & $\begin{array}{l}51 \% \text { a } 4.6 \text { anni } \\
\text { Stone Rate } 0.20\end{array}$ & $\begin{array}{l}- \\
-\end{array}$ & $\begin{array}{l}\text { Kohri K, } \\
\text { Urology 1990; 36: } \\
\text { 309-314 }\end{array}$ \\
\hline $\begin{array}{l}\text { Recidiva di calcoli } \\
\text { e Stone Rate }\end{array}$ & $\begin{array}{l}\text { Produttori recedivi } \\
\text { di calcoli di } \mathrm{CaOx} \\
\text { Maschi e Femmine }\end{array}$ & $\begin{array}{l}\text { Diuretici tiazidici } \\
\text { (Clortalidone) }\end{array}$ & $\begin{array}{l}13 \% \text { a } 3 \text { anni } \\
\text { Stone Rate } 0.05\end{array}$ & $\begin{array}{l}- \\
-\end{array}$ & $\begin{array}{l}\text { Ettinger B, } \\
\text { J Urol 1988; 139: 679- } \\
684\end{array}$ \\
\hline $\begin{array}{l}\text { Recidiva di calcoli } \\
\text { e Stone Rate }\end{array}$ & $\begin{array}{l}\text { Produttori recidivi } \\
\text { di calcoli di CaOx } \\
\text { con normocalciuria } \\
\text { e iperuricosuria } \\
\text { Maschi e Femmine }\end{array}$ & Allopurinolo & $\begin{array}{l}31 \% \text { a } 3 \text { anni } \\
\text { Stone Rate } 0.12\end{array}$ & $\begin{array}{l}- \\
-\end{array}$ & $\begin{array}{l}\text { Ettinger B, } \\
\text { NEJM 1986; } 315: 1386\end{array}$ \\
\hline
\end{tabular}


TABELLA I - DATI NECESSARI PER UN CALCOLO DELLA DIMENSIONE DEL CAMPIONE SULLA BASE DI TUTTI GLI INTERVENTIONAL RCT PUBBLICATI

\begin{tabular}{|c|c|c|c|c|c|}
\hline Obiettivo & Popolazione studiata & Intervento & Central tendency & Variabilità & Bibliografia \\
\hline $\begin{array}{l}\text { Recidiva di calcoli } \\
\text { e Stone Rate }\end{array}$ & $\begin{array}{l}\text { Produttori recidivi } \\
\text { di calcoli di } \mathrm{CaOx} \\
\text { Maschi e Femmine }\end{array}$ & $\begin{array}{l}\text { Diuretici tiazidici } \\
\text { (Clortalidone) }\end{array}$ & $\begin{array}{l}23 \% \text { a } 2.4 \text { anni } \\
\text { Stone Rate } 0.06\end{array}$ & - & $\begin{array}{l}\text { Ettinger B, In: Urolithia- } \\
\text { sis and Related Clinical } \\
\text { Research. Ed. Schwille } \\
\text { et al, Plenum Press, } \\
\text { 1985: pag 549-552 }\end{array}$ \\
\hline Stone Rate & $\begin{array}{l}\text { Produttori recidivi } \\
\text { di calcoli di calcio } \\
\text { Maschi e Femmine }\end{array}$ & Ortofosfato & 0.20 & - & $\begin{array}{l}\text { Robertson WG, In: } \\
\text { Urolithiasis and Related } \\
\text { Clinical Research. Ed. } \\
\text { Schwille et al, Plenum } \\
\text { Press, 1985; pag 545- } \\
\text { 548 }\end{array}$ \\
\hline Recidiva di calcoli & $\begin{array}{l}\text { Produttori recidivi } \\
\text { di calcoli di calcio } \\
\text { Maschi e Femmine }\end{array}$ & $\begin{array}{l}\text { Diuretici tiazidici } \\
\text { (Idroclorotiazide) }\end{array}$ & $22 \%$ a 3 anni & $95 \% \mathrm{CI}$ & $\begin{array}{l}\text { Laerum E, } \\
\text { Acta Med Scand 1984; } \\
\text { 215: } 383-9\end{array}$ \\
\hline Recidiva di calcoli & $\begin{array}{l}\text { Produttori recidivi } \\
\text { di calcoli di calcio } \\
\text { Maschi e Femmine }\end{array}$ & Acqua & $5.3 \%$ a 2 anni & - & $\begin{array}{l}\text { Ulman A, } \\
\text { Presse Medicale 1984; } \\
\text { 13: } 1257-1260\end{array}$ \\
\hline $\begin{array}{l}\text { Raddoppiamento dell'area dei } \\
\text { calcoli e recidiva di calcoli } \\
\text { (nuovi calcoli) }\end{array}$ & $\begin{array}{l}\text { Produttori di calcoli } \\
\text { di struvite con infezioni } \\
\text { da batteri che scindono } \\
\text { líurea } \\
\text { Maschi e Femmine }\end{array}$ & $\begin{array}{l}\text { Acido acetoidrossa- } \\
\text { mico }\end{array}$ & $\begin{array}{l}0 \% \text { a } 1.3 \text { anni } \\
0 \% \text { a } 1.3 \text { anni }\end{array}$ & - & $\begin{array}{l}\text { Williams JJ, } \\
\text { NEJM 1984; 311: 760- } \\
764\end{array}$ \\
\hline $\begin{array}{l}\text { Recidiva di calcoli } \\
\text { Calcio urinario }\end{array}$ & $\begin{array}{l}\text { Produttori recidivi } \\
\text { di calcoli di calcio } \\
\text { Maschi e Femmine }\end{array}$ & $\begin{array}{l}\text { Diuretici tiazidici } \\
\text { (Idroclorotiazide) }\end{array}$ & $\begin{array}{l}24 \% \text { a } 1 \text { anno } \\
\text { Ca } 153 \mathrm{mg} \\
\text { Ca } 2.28 \mathrm{mmol}\end{array}$ & $\begin{array}{l}- \\
\text { SD } 110 \\
\text { SD } 1.45\end{array}$ & $\begin{array}{l}\text { Scholz D, } \\
\text { J Urol 1982; 128: 903- } \\
907\end{array}$ \\
\hline $\begin{array}{l}\text { Recidiva di calcoli e Tasso di } \\
\text { recidiva (numero di calcoli/ } \\
\text { paziente/anno) }\end{array}$ & $\begin{array}{l}\text { Produttori recidivi } \\
\text { di calcoli di calcio } \\
\text { Maschi e Femmine }\end{array}$ & $\begin{array}{l}\text { Diuretici tiazidici } \\
\text { (Bendroflumetiazide) }\end{array}$ & $\begin{array}{l}15 \% \text { a } 1.5 \text { anni } \\
\text { Stone rate } 0.09\end{array}$ & - & $\begin{array}{l}\text { Brocks P, } \\
\text { Lancet 1981; 18: 124-125 }\end{array}$ \\
\hline $\begin{array}{l}\text { Recidiva di calcoli } \\
\text { e Calcio Urinario }\end{array}$ & $\begin{array}{l}\text { Produttori recidivi di } \\
\text { calcoli di CaOx in dieta a } \\
\text { basso contenuto di calcio } \\
\text { Maschi e Femmine }\end{array}$ & $\begin{array}{l}\text { Dieta a basso } \\
\text { contenuto di calcio }\end{array}$ & $\begin{array}{l}30 \% \text { a } 3 \text { anni } \\
\text { Ca } 183 \mathrm{mg}\end{array}$ & $\overline{\mathrm{SD}} 43$ & $\begin{array}{l}\text { Ettinger B, } \\
\text { Am J Med 1976; 61: } \\
\text { 200-206 }\end{array}$ \\
\hline Recidiva di calcoli & $\begin{array}{l}\text { Produttori di calcoli di } \\
\text { CaOx al primo episodio } \\
\text { Maschi e Femmine }\end{array}$ & $\begin{array}{l}\text { Dieta a basso } \\
\text { contenuto di proteine } \\
\text { animali e ad alto } \\
\text { contenuto di fibre, } \\
\text { normocalcica }\end{array}$ & $\begin{array}{l}\text { Tasso di recidiva } \\
7.1 / 100 \text { persone/anno }\end{array}$ & - & $\begin{array}{l}\text { Hiatt RA, } \\
\text { Am J Epidem 1996; } \\
\text { 144: } 25-33\end{array}$ \\
\hline Recidiva di calcoli & $\begin{array}{l}\text { Produttori recidivi } \\
\text { di calcoli di } \mathrm{CaOx} \\
\text { Maschi e Femmine }\end{array}$ & Allopurinolo & $\begin{array}{l}22 \% \text { a } 1 \text { anno } \\
25 \% \text { a } 5 \text { anni }\end{array}$ & - & $\begin{array}{l}\text { Smith MJV, J Urol 1977; } \\
\text { 117: 690-692 }\end{array}$ \\
\hline Recidiva di calcoli & $\begin{array}{l}\text { Produttori recidivi } \\
\text { di calcoli di calcio } \\
\text { Maschi e Femmine }\end{array}$ & $\begin{array}{l}\text { Diuretici tiazidici } \\
\text { (Idroclorotiazide) }\end{array}$ & $32 \%$ a 3 anni & - & $\begin{array}{l}\text { Fernandez-Rodriguez } \\
\text { M, Actas Urol Esp 2006; } \\
\text { 30: 305-309 }\end{array}$ \\
\hline
\end{tabular}

TRI= Tiselius Risk Index; SD= Deviazione Standard; IQR= Range Inter Quantile; RS= Supersaturazione Relativa; CaOx= Ossalato di Calcio; NAE= Escrezione di acido netta; Recidiva di calcoli=\% di pazienti che recidivano nel corso dell'intervento; Stone Rate= Numero di calcoli/paziente/anno; CI= Intervallo di Confidenza . 
TABELLA II - CARATTERISTICHE DI BASE DA RACCOGLIERE PER OGNI CALCOLOTICO ARRUOLATO IN UN INTERVENTIONAL RCT, AL FINE DI STABILIRE L'EFFETTO DELLA TERAPIA SIA FARMACOLOGICA CHE NON FARMACOLOGICA NELLA PREVENZIONE DELLE RECIDIVE DI CALCOLOSI

\section{INFORMAZIONI DI BASE OBBLIGATORIE}

Età

Sesso

Peso corporeo

Altezza

Pressione arteriosa

Storia familiare di calcoli

Composizione dei calcoli

Numero totale di calcoli formati

Età del primo episodio

Tasso di recidiva (numero di calcoli/paziente/anno)

Numero di calcoli ritenuti

Numero di procedure urologiche per eliminare

i calcoli
Malattie rilevanti concomitanti

Terapie farmacologiche concomitanti

Abitudini dietetiche (fondamentali per i trials sul trattamento dietetico)

Creatinina sierica

Calcio sierico

Acido urico sierico

Esame delle urine standard

Urocoltura (essenziale per i trials sui calcoli di struvite)

INFORMAZIONI DI BASE MOLTO UTILI

Sodiemia

Cloremia

Potassiemia

Magnesiemia

Fosforemia

PTH sierico

Bicarbonatemia
Volume delle urine nelle 24 ore

Creatinina urinaria delle 24 ore

$\mathrm{pH}$ urinario delle 24 ore

Calcio urinario delle 24 ore

Ossalato urinario delle 24 ore

Acido Urico urinario delle 24 ore

Citrato urinario delle 24 ore

Ammonio urinario delle 24 ore (per trials sui calcoli di struvite)

Screening per la cistina o cistina delle 24 ore (per trials sui calcoli di cistina)

INFORMAZIONI DI BASE ACCESSORIE

Urea urinaria delle 24 ore

Sodio urinario delle 24 ore

Cloro urinario delle 24 ore

Potassio urinario delle 24 ore

Fosforo urinario delle 24 ore
Magnesio urinario delle 24 ore
Solfato urinario delle 24 ore
Saturazione relative per: - $\mathrm{CaOx}$
- $\mathrm{CaP}$
- Acido Urico

si basano su un adeguato background scientifico. Considerando la letteratura, è utile notare che, attualmente, per quanto riguarda la nefrolitiasi, non è eticamente accettabile effettuare qualsiasi intervento senza che venga imposto anche un adeguato aumento dell'apporto di liquidi.

I farmaci concomitanti sono un punto cruciale: dovrebbero essere evitati tutti i farmaci, non previsti dal protocollo, che possono esercitare un effetto positivo o negativo sulla storia naturale della nefrolitiasi. La supplementazione con calcio, vitamina $\mathrm{C}$, vitamina $\mathrm{D}$ ecc. è accettabile solo se è equilibrata nei gruppi o se è stata pianificata in anticipo un'analisi per sottogruppi.

\section{Randomizzazione}

La randomizzazione rimuove i pregiudizi del ricercatore ad assegnare i trattamenti e produce gruppi che sono comparabili per fattori prognostici. Esistono diversi metodi di randomizzazione e tutti si basano sul presupposto che né il partecipante né lo sperimentatore sappiano quale trattamento sarà assegnato prima dell'inserimento dei partecipanti nello studio.

Metodi particolari di randomizzazione possono essere la randomizzazione stratificata, a blocchi e la adattativa. La stratificata è utile quando è prevista l'analisi per sottogruppi e in caso di sperimentazioni multicentriche in cui 


\section{TABELLA III - RCT CON “RECIDIVA” COME ENDPOINT PRIMARIO}

\begin{tabular}{|c|c|c|c|c|c|c|}
\hline Bibliografia & $\begin{array}{l}\text { Popolazione } \\
\text { nello studio }\end{array}$ & $\begin{array}{c}\text { No. di } \\
\text { pazienti che } \\
\text { hanno } \\
\text { completato } \\
\text { lo studio }\end{array}$ & Tipo di intervento & $\begin{array}{c}\text { Durata } \\
\text { media del } \\
\text { trattamento }\end{array}$ & Recidiva \% & $\mathbf{p}$ \\
\hline \multicolumn{7}{|l|}{ CALCIUM STONES } \\
\hline Borghi L. et al, 2002 & $\begin{array}{l}\text { Produttori di calcoli } \\
\text { di CaOx con ipercalciuria } \\
\text { idiopatica } \\
\text { Maschi }\end{array}$ & 103 & $\begin{array}{l}\text { Dieta a contenuto normale } \\
\text { di calcio e basso contenuto } \\
\text { di proteine e sale } \\
\text { vs } \\
\text { Dieta tradizionale a basso } \\
\text { contenuto di calcio }\end{array}$ & 5 anni & $20 \%$ vs $38 \%$ & $\mathrm{p}=0.04$ \\
\hline \multirow[b]{2}{*}{ Soygur T. et al, 2002} & \multirow{2}{*}{$\begin{array}{l}\text { Produttori di calcoli } \\
\text { di CaOx post-litotrissia, } \\
\text { sia senza calcoli che } \\
\text { con frammenti residui } \\
\text { Maschi e Femmine }\end{array}$} & \multirow[b]{2}{*}{60} & \multirow{2}{*}{$\begin{array}{l}\text { KCitrato } 60 \mathrm{mEq} \\
\text { vs } \\
\text { Nessun trattamento }\end{array}$} & \multirow[b]{2}{*}{1 anno } & $\begin{array}{l}0 \% \text { vs } 28.5 \% \\
\text { (senza calcoli) }\end{array}$ & $\mathrm{p}<0.05$ \\
\hline & & & & & $\begin{array}{l}12.5 \% \text { vs } 44.5 \% \\
\text { (frammenti } \\
\text { residui) }\end{array}$ & $\mathrm{p}<0.05$ \\
\hline Ettinger B. et al, 1997 & $\begin{array}{l}\text { Produttori recidivi } \\
\text { di calcoli di } \mathrm{CaOx} \\
\text { Maschi e Femmine }\end{array}$ & 41 & $\begin{array}{l}\text { K-Mg-Citrato } \\
(42-21-63 \mathrm{mEq}) \\
\text { vs } \\
\text { Placebo }\end{array}$ & 3 anni & $12.9 \%$ vs $63.6 \%$ & Non eseguito \\
\hline Borghi L. et al, 1996 & $\begin{array}{l}\text { Produttori di calcoli } \\
\text { (primo episodio) } \\
\text { Maschi e Femmine }\end{array}$ & 199 & $\begin{array}{l}\text { Apporto di acqua tale da } \\
\text { raggiungere un volume } \\
\text { urinario }>2 \mathrm{~L} \\
v s \\
\text { Nessun trattamento }\end{array}$ & 5 anni & $12.1 \%$ vs $27 \%$ & $\mathrm{p}=0.008$ \\
\hline Hofbauer J. et al, 1994 & $\begin{array}{l}\text { Produttori recidivi } \\
\text { di calcoli di CaOx } \\
\text { Maschi e Femmine }\end{array}$ & 22 & $\begin{array}{l}\text { Assunzione di Na-K-Citrato } \\
\text { x3 tale da ottenere un } \mathrm{pH} \\
\text { di } 7-7.2 \\
\text { vs } \\
\text { Raccomandazioni } \\
\text { profilattiche generali }\end{array}$ & 3 anni & $69 \%$ vs $73 \%$ & $\mathrm{p}=0.65$ \\
\hline Barcelo P. et al, 1993 & $\begin{array}{l}\text { Produttori recidivi } \\
\text { di calcoli di CaOx e CaP } \\
\text { con ipocitraturia } \\
\text { Maschi e Femmine }\end{array}$ & 38 & $\begin{array}{l}\text { K Citrato } 20 \mathrm{mEq} \times 3 \\
\text { vs } \\
\text { Placebo }\end{array}$ & 3 anni & $27.8 \%$ vs $80 \%$ & Non eseguito \\
\hline Borghi L. et al, 1993 & $\begin{array}{l}\text { Produttori recidivi di } \\
\text { calcoli di } \mathrm{CaOx} \text { e } \mathrm{CaOx}- \\
\mathrm{CaP} \text { con ipercalciuria, } \\
\text { senza calcoli al momento } \\
\text { della valutazione di base }\end{array}$ & 64 & $\begin{array}{l}\text { Dieta e fluidi (I) } \\
v s \\
\text { Dieta e fluidi + Indapamide } \\
2.5 \mathrm{mg} \text { (II) } \\
v s \\
\text { Dieta e fluidi + Indapamide } \\
2.5 \mathrm{mg}+\text { Allopurinolo } \\
300 \mathrm{mg} \text { (III) }\end{array}$ & 3 anni & $\begin{array}{l}42.8 \% \text { (I) } \\
\text { vs } \\
15.8 \% \text { (II) } \\
\text { vs } \\
12.5 \% \text { (III) }\end{array}$ & $\begin{array}{c}\mathrm{p}=0.02 \\
\text { (II e III } v s \mathrm{I} \text { ) }\end{array}$ \\
\hline Ohkawa M. et al, 1992 & $\begin{array}{l}\text { Produttori recidivi di } \\
\text { calcoli di CaOx e CaOx- } \\
\text { CaP con ipercalciuria } \\
\text { Maschi e Femmine }\end{array}$ & 175 & $\begin{array}{l}\text { Triclormetiazide } \\
4 \mathrm{mg} \\
v s \\
\text { Nessuna terapia }\end{array}$ & 2 anni & $8.3 \%$ vs $14.1 \%$ & $0.05<\mathrm{p}>0.1$ \\
\hline
\end{tabular}


TABELLA III - RCT CON “RECIDIVA” COME ENDPOINT PRIMARIO

\begin{tabular}{|c|c|c|c|c|c|c|}
\hline Bibliografia & $\begin{array}{c}\text { Popolazione nello } \\
\text { studio }\end{array}$ & $\begin{array}{c}\text { No. di } \\
\text { pazienti che } \\
\text { hanno } \\
\text { completato } \\
\text { lo studio }\end{array}$ & Tipo di intervento & $\begin{array}{c}\text { Durata } \\
\text { media del } \\
\text { trattamento }\end{array}$ & Recidiva \% & $\mathbf{p}$ \\
\hline Ettinger B. et al, 1988 & $\begin{array}{l}\text { Produttori recidivi } \\
\text { di calcoli di } \mathrm{CaOx} \\
\text { Maschi e Femmine }\end{array}$ & 124 & $\begin{array}{l}\text { Placebo } \\
\text { vs } \\
\text { Latte di Magnesia } \\
325 \mathrm{mg} \times 2 \\
\text { (Gruppo A) } \\
\text { vs } \\
\text { Latte di Magnesia } \\
650 \mathrm{mg} \times 2 \\
\text { (Gruppo B) } \\
\text { vs } \\
\text { Clortalidone } 25 \mathrm{mg} \\
\text { (Gruppo C) } \\
\text { vs } \\
\text { Clortalidone } 50 \mathrm{mg} \\
\text { (Gruppo D) }\end{array}$ & 3 anni & $\begin{array}{l}45 \% \text { (Plac) } \\
v s \\
27 \% \text { (A) } \\
v s \\
33 \%(\mathrm{~B}) \\
v s \\
16 \%(\mathrm{C}) \\
v s \\
13 \%(\mathrm{D})\end{array}$ & $\begin{array}{l}\mathrm{p}<0.05 \\
\text { (Gruppo D vs } \\
\text { Altri) }\end{array}$ \\
\hline Ettinger B. et al, 1986 & $\begin{array}{l}\text { Produttori recidivi } \\
\text { di calcoli di CaOx } \\
\text { con normocalciuria } \\
\text { e iperuricosuria } \\
\text { Maschi e Femmine }\end{array}$ & 60 & $\begin{array}{l}\text { Allopurinolo } 100 \mathrm{mg} \times 3 \\
\text { vs } \\
\text { Placebo }\end{array}$ & 3 anni & $31 \%$ vs $58 \%$ & $\mathrm{p}<0.001$ \\
\hline Laerum E. et al, 1984 & $\begin{array}{l}\text { Produttori recidivi } \\
\text { di calcoli di calcio } \\
\text { in medicina generale } \\
\text { Maschi e Femmine }\end{array}$ & 48 & $\begin{array}{l}\text { Idroclorotiazide } \\
25 \mathrm{mg} \\
v s \\
\text { placebo }\end{array}$ & 3 anni & $22 \%$ vs $48 \%$ & $\mathrm{p}=0.05$ \\
\hline Ulman A. et al, 1984 & $\begin{array}{l}\text { Produttori recidivi } \\
\text { di calcoli di calcio } \\
\text { Maschi e Femmine }\end{array}$ & 51 & $\begin{array}{l}\text { Terapia idropinica (I) } \\
\text { vs } \\
\text { Terapia Idropinica + } \\
\text { Idroclorotiazide } \\
50 \mathrm{mg} \text { (II) } \\
\text { vs } \\
\text { Fosforo } 750 \mathrm{mg} \times 2 \text { (III) }\end{array}$ & 2 anni & $\begin{array}{l}5.3 \% \text { (I) vs } 26 \% \\
\text { (II) } \\
\text { [\% (III) n.d.] }\end{array}$ & $\mathrm{p}<0.05$ \\
\hline Scholz D. et al, 1982 & $\begin{array}{l}\text { Produttori recidivi } \\
\text { di calcoli di calcio } \\
\text { Maschi e Femmine }\end{array}$ & 48 & $\begin{array}{l}\text { Idroclorotiazide } \\
25 \mathrm{mg} \mathrm{x} 2 \\
v s \\
\text { Placebo }\end{array}$ & 1 anno & $24 \%$ vs $23 \%$ & $\begin{array}{l}\mathrm{p}=\mathrm{ns} \\
\text { (valori non } \\
\text { mostrati) }\end{array}$ \\
\hline Brocks P. et al, 1981 & $\begin{array}{l}\text { Produttori recidivi } \\
\text { di calcoli di calcio } \\
\text { Maschi e Femmine }\end{array}$ & 62 & $\begin{array}{l}\text { Bendroflumetiazide } 2.5 \times 3 \\
\text { vs } \\
\text { Placebo }\end{array}$ & 1.5 anni & $15 \%$ vs $17 \%$ & Non eseguito \\
\hline Ettinger B., 1976 & $\begin{array}{l}\text { Produttori recidivi } \\
\text { di calcoli di CaOx a dieta } \\
\text { a basso contenuto di sodio } \\
(600 \mathrm{mg})\end{array}$ & 71 & $\begin{array}{l}\text { K Acido Fosfato } \\
(1.4 \mathrm{~g} \text { di P) (I) } \\
\text { vs } \\
\text { Placebo (II) } \\
\text { vs } \\
\text { Dieta a basso contenuto } \\
\text { di calcio da sola (III) }\end{array}$ & 3 anni & $\begin{array}{l}50 \% \text { (I) } \\
v s \\
30 \% \text { (II) } \\
\text { vs } \\
53 \% \text { (III) }\end{array}$ & Non eseguito \\
\hline
\end{tabular}


TABELLA III - RCT CON “RECIDIVA” COME ENDPOINT PRIMARIO

\begin{tabular}{|c|c|c|c|c|c|c|}
\hline Bibliografia & $\begin{array}{l}\text { Popolazione } \\
\text { nello studio }\end{array}$ & $\begin{array}{c}\text { No. di } \\
\text { pazienti che } \\
\text { hanno } \\
\text { completato } \\
\text { lo studio }\end{array}$ & Tipo di intervento & $\begin{array}{c}\text { Durata } \\
\text { media del } \\
\text { trattamento }\end{array}$ & Recidiva \% & $\mathbf{p}$ \\
\hline Hiatt RA. et al, 1996 & $\begin{array}{l}\text { Produttori di calcoli } \\
\text { di CaOx al primo episodio }\end{array}$ & 99 & $\begin{array}{l}\text { Dieta a basso contenuto } \\
\text { di proteine animali e ad } \\
\text { elevato contenuto di fibre, } \\
\text { normocalcica + Terapia } \\
\text { Idropinica } \\
v s \\
\text { Dieta normocalcica + } \\
\text { Terapia Idropinica }\end{array}$ & 4.5 anni & $32 \%$ vs $5 \%$ & $\mathrm{p}=0.006$ \\
\hline Smith MJV, 1977 & $\begin{array}{l}\text { Produttori recidivi } \\
\text { di calcoli di } \mathrm{CaOx}\end{array}$ & 92 & $\begin{array}{l}\text { Allopurinolo } 100 \mathrm{mg} \\
\text { vs } \\
\text { Placebo }\end{array}$ & 1 anno & $22 \%$ vs $48 \%$ & Non eseguito \\
\hline $\begin{array}{l}\text { Fernandez-Rodriguez } \\
\text { A. et al, } 2006\end{array}$ & $\begin{array}{l}\text { Produttori recidivi } \\
\text { di calcoli di calcio }\end{array}$ & 150 & $\begin{array}{l}\text { Idroclorotiazide } 50 \mathrm{mg}+ \\
\text { Cloruro di potassio } \\
20 \mathrm{mEq} / \text { die (I) } \\
v s \\
\text { Idroclorotiazide } 50 \mathrm{mg} \text { (II) } \\
v s \\
\text { Nessuna terapia (III) }\end{array}$ & 3 anni & $\begin{array}{l}30 \% \text { (I) } \\
v s \\
32 \% \text { (II) } \\
v s \\
56 \% \text { (III) }\end{array}$ & $\mathrm{p}<0.05$ \\
\hline \multicolumn{7}{|c|}{ CALCOLI DI STRUVITE } \\
\hline Williams JJ, 1984 & $\begin{array}{l}\text { Produttori di calcoli } \\
\text { di struvite con infezioni } \\
\text { da organismi che scindono } \\
\text { l'urea } \\
\text { Maschi e Femmine }\end{array}$ & 33 & $\begin{array}{l}\text { Acido Acetoidrossamico } \\
15 \mathrm{mg} / \mathrm{kg} / \text { day } \\
\text { vs } \\
\text { Placebo }\end{array}$ & 1.3 anno & $0 \%$ vs $37 \%$ & $\mathrm{p}<0.01$ \\
\hline \multicolumn{2}{|c|}{ CALCOLI DI ACIDO URICO } & & & & & \\
\hline \multicolumn{7}{|l|}{ Nessun RCT disponibile } \\
\hline \multicolumn{2}{|c|}{ CALCOLI DI CISTINA } & & & & & \\
\hline
\end{tabular}

ogni strato è rappresentato da un centro. La randomizzazione a blocchi ha lo scopo di impedire lo squilibrio nel numero di pazienti assegnati a ciascun gruppo. I blocchi sono brevi sequenze al termine delle quali il numero di assegnazioni al trattamento è uniformemente distribuito (ad esempio, ABBA AB ABABBBAA). Nella randomizzazione adattativa viene modificata la probabilità di assegnazione man mano che lo studio prosegue (18). Può essere impiegata per correggere lo squilibrio delle caratteristiche di base per quegli studi che permettono l'arruolamento di un numero limitato di pazienti.

Nonostante l'importanza della randomizzazione, spesso gli RCT pubblicati sulla nefrolitiasi non forniscono dettagli circa i metodi utilizzati.

\section{Aderenza e compliance dei partecipanti}

\section{Monitoraggio della risposta}

Molti problemi di aderenza al protocollo possono essere prevenuti prima di arruolare i partecipanti. Una volta che un partecipante è arruolato, è fondamentale l'adozione di misure per motivarlo. 
Alcuni pazienti possono ritirarsi dallo studio perché non vogliono o non possono tornare alle visite di follow-up. Altri pazienti, assegnati al gruppo di controllo, potrebbero seguire il regime di intervento o viceversa. Altri apparentemente aderenti al trattamento assegnato, di fatto non rispettano le prescrizioni.

Per "persi al follow-up" si intende la perdita irrimediabile del contatto con il paziente, e quindi non c'è modo di valutare il suo risultato. Per "uscita dal trattamento" si intende il paziente che continua a presentarsi per le visite programmate, ma che non ha rispettato il trattamento assegnato. Alcuni Autori fanno riferimento a quest'ultima categoria con il termine di "drop-out". Per "ritiro dallo studio" si intende il paziente che non è disposto a continuare le visite in programma, ma può ancora essere contattato (per esempio, con una telefonata). Lo scenario peggiore è, ovviamente, quello del "persi al follow-up". È necessario ogni sforzo per evitare di avere anche un singolo paziente "perso al followup". È comunque possibile recuperare le informazioni sul ritiro senza invalidare i risultati dello studio, tuttavia è sicuramente meglio favorire il rispetto del protocollo, attraverso l'attuazione di strategie appropriate.

A causa della storia naturale e delle caratteristiche della malattia, è molto difficile nella nefrolitiasi ridurre al minimo i pazienti "ritirati": infatti, la nefrolitiasi colpisce di solito soggetti "sani" che aderiscono ai consigli medici al momento di un episodio acuto di colica, ma ben presto perdono interesse. Di conseguenza, il numero di drop-out in RCTs pubblicati sulla nefrolitiasi è notevole, in particolare per quelli con un lungo follow-up. Tra le strategie per minimizzare questo fenomeno, potrebbe essere utile pianificare visite periodiche, avere frequenti contatti telefonici, fornire materiale illustrativo, informare il Medico di Medicina Generale ed effettuare periodiche raccolte delle urine consentendo al paziente di vedere i risultati concreti del suo impegno. Anche l'esecuzione di un intervento semplice, agevole per il paziente, diminuisce il tasso di abbandono. In caso di intervento dietetico, è importante avere una valutazione effettuata da un dietologo. Inoltre, allinizio dello studio, prima della randomizzazione, può essere eseguito un periodo di followup, senza intervento, con l'obiettivo di valutare la compliance e le caratteristiche di base (periodo "run-in") $(6,9,11)$. Infine, è utile fornire alcuni "servizi", come ambulatori dedicati ed esami gratuiti evitando le liste di attesa.

\section{Valutazione dell'outcome ed endpoints surrogati}

Negli RCTs sulla nefrolitiasi, l'outcome primario dovrebbe essere la "recidiva”. E importante specificare il metodo impiegato per diagnosticare le recidive. Il caso più semplice è quando al primo episodio c'è un solo calcolo che viene espulso: in questa situazione, un nuovo episodio di colica o la comparsa di un nuovo calcolo asintomatico rilevato con tecniche di imaging, permettono la diagnosi. Se, invece, il primo episodio si presenta come un caso con più calcoli o calcoli ritenuti, allora colica non è sinonimo di recidiva, ma potrà essere diagnosticata solo se i calcoli sono aumentati in numero e dimensioni. Tuttavia, a causa della variabilità inter-osservatore, tale diagnosi spesso non è facile. Il radiologo dovrebbe comunque lavorare "in cieco".

La realizzazione dell'endpoint "ideale", vale a dire la recidiva, richiede un lungo periodo di follow-up. In mancanza di questo si possono valutare endpoints surrogati (19), che comunemente sono i fattori urinari di rischio litogeno, dal momento che lo squilibrio di tali fattori espone i pazienti ad un maggiore tasso di recidiva. Vari studi hanno dimostrato che i fattori di rischio urinari, per esempio basso volume urinario e alti livelli di calciuria, sono associati con intervallo di recidiva più breve (20).

Tuttavia, oltre ad essere endpoints surrogati, gli indici urinari presentano anche lo svantaggio di essere multipli con necessità di confronti statistici multipli con conseguente aumento della possibilità di incorrere in falsi risultati positivi.

\section{Analisi dei dati}

\section{Analisi per sottogruppi}

Una tecnica di analisi è quella di dividere la popolazione in studio in sottogruppi in modo da consentire il confronto tra controllo e intervento all'interno di ogni sottogruppo. L'obiettivo è quello di individuare le categorie con più probabilità di trarre beneficio dal trattamento. Grande cautela deve essere esercitata nell'esecuzione di tali analisi, perché portano a test multipli con un aumento della probabilità di risultati falsi-positivi, e a una perdita di potenza che porta a falsi risultati negativi (21). Il tipo più debole di analisi per sottogruppi sono le analisi "post-hoc" cioè, le analisi suggerite dai dati stessi. L'analisi per sottogruppi dovrebbe essere prevista prima di iniziare lo studio.

Un esempio di analisi per sottogruppi utile nella calcolosi riguarda la suddivisione per sesso, gravità della malattia e tipo di calcoli, soprattutto quando la popolazione in studio è eterogenea. Ad esempio, nel nostro RCT sugli effetti di una dieta normal calcium, low salt, low protein in maschi ipercalciurici recidivanti, in base al presupposto che la dieta non può essere efficace su calcoli già in formazione, abbiamo effettuato un'analisi per sottogrup- 
pi selezionando gli uomini ad alto rischio, cioè con una storia di cinque o più episodi di coliche l'anno o con più di 10 calcoli. Questo ci ha permesso di valutare che il trattamento dietetico è stato poco efficace nei pazienti più gravi (9). Inoltre, in studi randomizzati che testavano l'effetto profilattico dell'allopurinolo sulle recidive, l'analisi per sottogruppi ha mostrato una maggiore riduzione del tasso di recidiva negli iperuricosurici rispetto ai non iperuricosurici $(11,16)$.

\section{Tipi peculiari di disegno dello studio}

\section{Lo studio cross-over}

Il disegno cross-over, eliminando l'errore dovuto alla variabilità tra i pazienti, richiede un minor numero di soggetti da arruolare rispetto al disegno a gruppi paralleli. I soggetti effettuano in sequenza tutti i trattamenti. Lipotesi alla base è che gli effetti del primo intervento non si riverberino sul secondo. Per essere sicuri di questa ipotesi, è necessario eseguire un adeguato periodo di wash-out. Inoltre, se tutti i soggetti ricevono i due trattamenti nello stesso ordine, le differenze osservate tra i trattamenti potrebbero essere confuse con altre modifiche che avvengono naturalmente nel corso del tempo. Il "two-period, two treatment cross-over trial" supera questa difficoltà, avendo la metà dei soggetti che ricevono un trattamento A seguito da un trattamento $\mathrm{B}$, mentre l'altra metà riceve $\mathrm{B}$ seguito da $\mathrm{A}(22)$.

In studi di intervento sulla nefrolitiasi, se l'endpoint primario è lo "stone rate", il protocollo cross-over non è applicabile. Invece, questo tipo di studio è utile per valutare l'effetto di interventi sui fattori di rischio urinari (23-27).

\section{Additività e sinergia: lo studio fattoriale}

A volte il problema potrebbe sorgere dal fatto che due diversi trattamenti esercitano un effetto additivo o sinergico sul rischio di calcolosi. Questo problema è meglio esaminato da uno studio "fattoriale", in cui ogni braccio di trattamento rappresenta una di tutte le possibili combinazioni dei trattamenti in fase di test (28). Immaginiamo, per esempio, uno studio fattoriale in cui i calcolotici sono randomizzati a diuretico tiazidico o KCitrato e dove l'outcome è la recidiva. Il design $2 \mathrm{x} 2$ implicherebbe quattro gruppi di trattamento vale a dire, diuretico e Kcitrato, diuretico e Placebo, Placebo e Kcitrato e Placebo Placebo. L'additività si verifica quando l'effetto congiunto dei risultati di due trattamenti è la somma dei loro effetti separati. Il sinergismo si verifica quando l'effetto congiunto dei due trattamenti è maggiore di quanto ci si aspetterebbe dalla somma dei loro effetti.

\section{Lo studio dose-risposta}

Lo studio valuta quale è la dose più efficace di intervento da somministrare. Di conseguenza, i pazienti sono assegnati al braccio placebo, oppure a gruppi corrispondenti ciascuno a livelli distinti di dose di farmaco o di una sostanza nutritiva. Questo è un disegno potente, nel senso che non solo rafforza gli elementi di prova circa l'efficacia del trattamento, ma fornisce anche informazioni circa lintervento minimo efficace. Quest'ultimo aspetto è particolarmente importante quando il trattamento viene mal tollerato dai pazienti. Per fare un esempio, sarebbe utile questo disegno sperimentale per valutare l'effetto di vari livelli di assunzione di $\mathrm{NaCl}$ sul tasso di recidiva di pazienti con calcolosi calcica.

\section{Conclusioni}

Le norme generali per un corretto RCT (2), si applicano anche alla nefrolitiasi, e cioè, popolazione di adeguate dimensioni, follow-up sufficiente, definizione precisa dellintervento, risultati valutati in modo analogo, informazioni mancanti ridotte al minimo e principio "intention-to-treat" per l'analisi dei dati. Tuttavia, in molti studi pubblicati queste regole spesso non sono state rispettate certamente non per negligenza, ma a causa delle difficoltà inerenti alla storia naturale della calcolosi renale, una malattia che colpisce soggetti "sani" che diventano poco interessati a trattamenti preventivi una volta che il dolore acuto della colica è diventato un ricordo remoto. Nonostante la prevalenza relativamente elevata della malattia, è sorprendente notare nella letteratura medica la mancanza di studi clinici di grandi dimensioni, in particolare nella Medicina Generale. Questo può essere in parte attribuito alla mancanza di interesse da parte dell'industria farmaceutica, infatti la nefrolitiasi può essere considerata quasi una malattia "orfana" di farmaci. Per queste ragioni, è auspicabile che i ricercatori "istituzionali” facciano ogni sforzo per colmare questa lacuna.

Indirizzo degli Autori:

Prof. Loris Borghi

Dip. Scienze Cliniche

Università di Parma

Via A. Gramsci 14

43126 Parma, Italy

loris.borghi@unipr.it 


\section{Bibliografia}

1. Miettinen OS. The modern scientific physician: 5 . the useful property of an intervention. CMAJ 2001;165: 1059-60.

2. Altman DG, Schulz KF, Moher D, et al for the CONSORT Group. The revised CONSORT statement for reporting randomized trials: explanation and elaboration. Ann Intern Med 2001; 134: 663-94.

3. Gross CP, Mallory R, Heiat A, et al. Reporting the recruitment process in clinical trials: who are these patients and how did they get there? Ann Intern Med 2002; 137: 10-6.

4. Laerum $\mathrm{E}$ and Larsen S. Thiazide prophylaxis of urolithiasis. A double-blind study in general practice. Acta Med Scand 1984; 215: 383-9.

5. Borghi L, Meschi T, Guerra A, et al. Randomized prospective study of a nonthiazide diuretic, indapamide, in preventing calcium stone recurrences. J Cardiovasc Pharm 1993; 22(Suppl): S78-S86.

6. Ettinger B, Citron JT, Livermore B, et al. Chlorthalidone reduces calcium oxalate calculous recurrence but magnesium hydroxide does not. J Urol 1988; 139: 679-84.

7. Ohkawa M, Tokunaga S, Nakashima T. et al. Thiazide treatment for calcium urolithiasis in patients with idiopathic hypercalciuria. Br J Urol 1992; 69: 571-6.

8. Barcelo P, Wuhl O, Servitge E, et al. Randomized doubleblind study of potassium citrate in idiopathic hypocitraturic calcium nephrolithiasis. J Urol 1993; 150: 1761-4.

9. Borghi L, Schianchi T, Meschi T, et al. Comparison of two diets for the prevention of recurrent stones in idiopathic hypercalciuria. N Engl J Med 2002; 346: 77-84.

10. Ettinger B, Citron JT, Tang A, et al. Prophylaxis of calcium oxalate stones: clinical trials of allopurinol, magnesium hydroxide and chlorthalidone In: Urolithiasis and Related Clinical Research. Ed. Schwille et al. Plenum Press, 1985; 549-52.

11. Ettinger B, Tang A, Citron JT, et al. Randomized trial of allopurinol in the prevention of calcium oxalate calculi. $\mathrm{N}$ Engl J Med 1986; 315: 1386-9.

12. Goldfarb DS, Modersitzki F, Asplin JR. A randomized, controlled trial of lactic acid bacteria for idiopathic hyperoxaluria. Clin J Am Soc Nephrol 2007; 2: 745-9.

13. Lachin JM. Introduction to sample size determination and power analysis for clinical trials. Control Clin Trials 1981; 2: 93-113.

14. Borghi L, Meschi T, Amato F, et al. Urinary volume, wa- ter and recurrences in idiopathic calcium nephrolithiasis: a 5-year randomized prospective study. J Urol 1996; 155 : 839-43.

15. Ettinger B, Pak CY, Citron JT, et al. Potassium-magnesium citrate is an effective prophylaxis against recurrent calcium oxalate nephrolithiasis. J Urol 1997; 158: 2069-73.

16. Kohri K, Kodama M, Katayama Y, et al . Allopurinol and thiazide effects on new urinary stone formed after discontinued therapy in patients with urinary stones. Urology 1990; 36: 309-14.

17. Robertson WG, Peacock M, Selby PL, et al. A multicentre trial to evaluate three treatments for recurrent idiopathic calcium stone disease - a preliminary report Robertson WG. In: Urolithiasis and Related Clinical Research. Ed. Schwille, et al. Plenum Press, 1985; 545-8.

18. Scott NW, McPherson GC, Ramsay CR, et al. The method of minimization for allocation to clinical trials: a review. Control Clin Trials 2002; 23: 662-74.

19. Zerwekh JE, Odvina CV, Wuermser LA, et al. Reduction of renal stone risk by potassium-magnesium citrate during 5 weeks of bed rest. J Urol 2007; 177: 2179-84.

20. Strauss AL, Coe FL, Deutsch L, et al. Factors that predict relapse of calcium nephrolithiasis during treatment: a prospective study. Am J Med 1982; 72: 17-24.

21. Lagakos SW. The challenge of subgroup analyses - reporting without distorting. N Engl J Med 2006; 354: 16.

22. Hills M and Armitage P. The two-period cross-over clinical trial. Br J Clin Pharmacol 1979; 8: 7-20.

23. Domrongkitchaiporn S, Sopassathit W, Stitchantrakul W, et al. Schedule of taking calcium supplement and the risk of nephrolithiasis. Kidney Int 2004; 65: 1835-41.

24. Heller HJ, Doerner MF, Brinkley LJ, Adams-Huet B, Pak CY. Effect of dietary calcium on stone forming propensity. J Urol 2003; 169(2): 470-4.

25. Massey LK, Liebman M, Kynast-Gales SA. Ascorbate increases human oxaluria and kidney stone risk. J Nutr 2005; 135: 1673-7.

26. McHarg T, Rodgers A, Charlton K. Influence of cranberry juice on the urinary risk factors for calcium oxalate kidney stone formation. BJU Int 2003; 92: 765-8.

27. Odvina CV. Comparative value of orange juice versus lemonade in reducing stone-forming risk. Clin J Am Soc Nephrol 2006; 1: 1269-74.

28. Fleiss JL Factorial experiments. In: The design and analysis of clinical experiments. New York: John Wiley and Sons, 1986. 